\title{
Analysis of bed occupancy data on the acute medical unit
}

\author{
Authors: Danil Baykov, ${ }^{\mathrm{A}}$ Anne Skeldon ${ }^{\mathrm{A}}$ and Martin Whyte ${ }^{\mathrm{A}}$
}
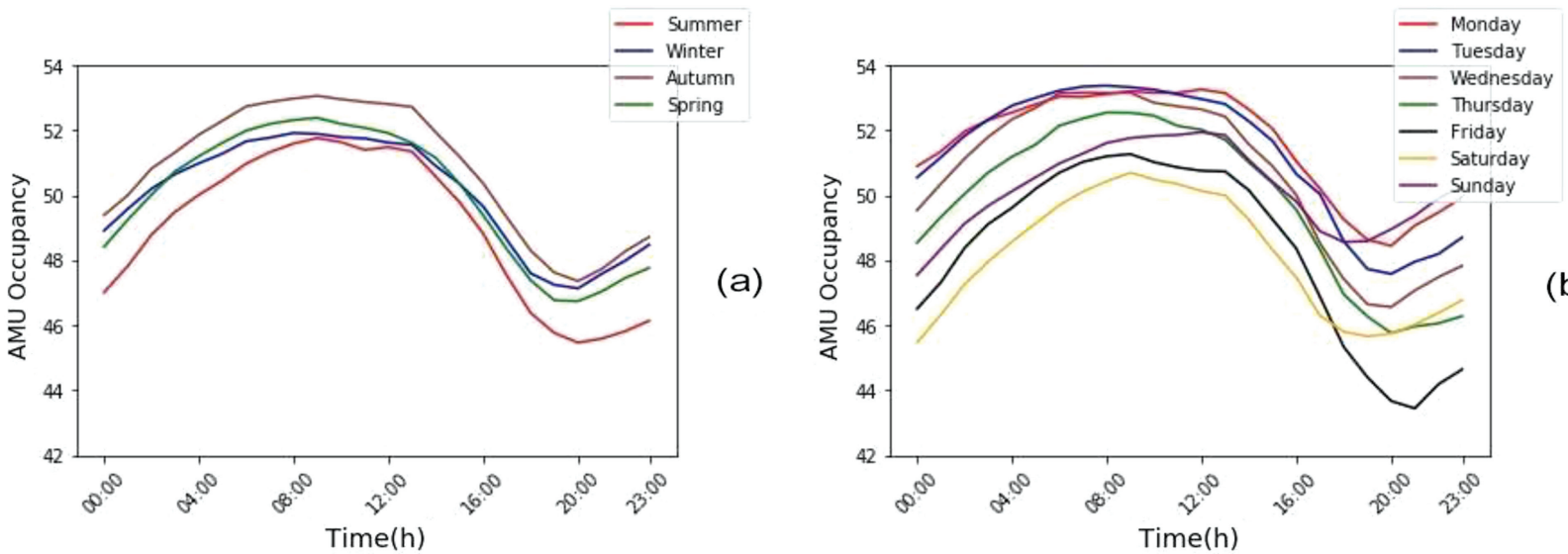

(b)

Fig 1. a) Occupancy follows the same daily pattern for different seasons, but is lower during summer (1 June - 31 August) than in winter (1 December - 28 February). b) Occupancy follows the same daily pattern for different days of the week, but is lower at the weekends.

\section{Introduction}

Hospitals may struggle to operate effectively and meet quality standards when unscheduled (emergency) admission rates are high in relation to operational capacity. Understanding patterns of ward admission and discharge from acute medical units (AMU) can help inform resource allocation. Discharges early in the day are advocated as a means to improve bed management, but delays may arise from post-AMU bed availability. It is unclear whether any change in AMU discharge patterns pre-dates hospital-wide pressure or whether it is a reaction to it.

\section{Materials and methods}

Anonymous data from 1 Jan 2016 to 31 December 2017 comprising the time and date of admission and discharge from a 56-bed AMU at a large, urban tertiary hospital were collected. For a measure of 'pressure' in the hospital system, we collected the daily (midnight) hospital occupancy by medical inpatients, as well as daily numbers of medical patients on 'outlying' (non-medical) wards over the same timeframe.

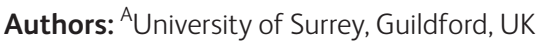

Admission and discharge times for the AMU were used to determine AMU occupancy as a function of time of day. In order to capture how daily patterns varied with day of the week and with season, data were then averaged. In order to investigate whether there was a correlation between the timing of peak daily occupancy and 'pressure' in the hospital system, the time of day at which maximum occupancy occurred was also calculated.

\section{Results and discussion}

In total there were 18,158 patient episodes. Bed occupancy followed a strictly cyclical circadian rhythm (nadir early evening, peak early morning), within a circaseptan rhythm (lower at weekends), within an annual rhythm, see Fig 1. Although occupancy was lower in summer, the mean daily amplitude of the occupancy rhythm was higher in summer than in winter (6.3 vs 4.8 ) and occurred later.

The average patterns mask the high daily variability in occupancy patterns. Although time of peak occupancy typically occurs around 08:00, it can occur at any point in the day, as shown in Fig 2. Initial results comparing AMU occupancy with overall hospital pressure do not suggest that there is a strong correlation between the time of peak daily AMU occupancy with overall hospital pressure, but 

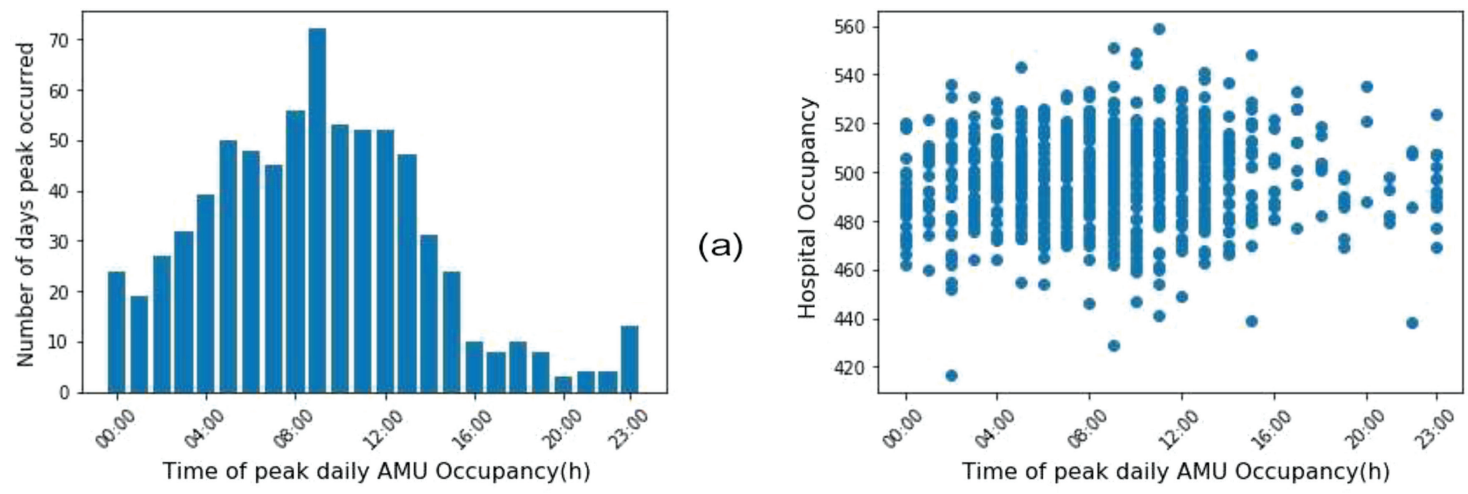

(b)

Fig 2. Time of peak occupancy. a) Histogram of time of day of peak AMU occupancy. b) Hospital occupancy vs time of day of peak AMU occupancy.

further work needs to be done to investigate daily and seasonal differences and time lags.

\section{Conclusion}

Hospital bed occupancy may be better modelled using circadian mathematical techniques. If a change in bed policy is made, this could be better tested via its effect on the 'circadian system'.

\section{Conflicts of interest}

None declared. 